\title{
Clinicopathological Evaluation of Abnormal Uterine Bleeding with Special Reference to Estrogen Receptor (ER), Progesterone Receptor (PR)2 and HER-2 / NEU Status
}

\author{
Tanushree Satpathy ${ }^{1 *}$, Binapani Satpathy ${ }^{2}$ and Prasanna Kumar Satpathy ${ }^{3}$ \\ ${ }^{1}$ Dept. of Pathology, GITAM Institute of Medical sciences and research, Vishakhapatnam, India \\ ${ }^{2}$ Dept. Obstetrics and Gynecology, Hi-Tech Medical College, Bhubaneswar, India \\ ${ }^{3}$ Dept. of Pathology, Hi-Tech Medical College, Bhubaneswar, India
}

\begin{abstract}
Background: Abnormal Uterine Bleeding (AUB) may be defined as any variation from normal menstrual cycle including alteration in its regularity, frequency, duration of flow and amount of blood loss. AUB can present as menorrhoea, metrorrhagia, poly menorrhoea and dysfunctional uterine bleeding. The study aimed to found out the various aetiopathological factors responsible for AUB. To correlate clinical presentation with histopathological examination and also to study the hormone receptor markers ER (Estrogen Receptors), PR (Progesterone receptor) and marker of oncogene HER-2/NEU for prognostication.

Methods: This study was conducted during the period Sept. 2012 to January 2015 in the Department of Obstetrics and Gynecology in collaboration with Dept. of Pathology, Hi-Tech Medical College, Bhubaneswar. 140 patients of 15-55 yrs of age groups were randomly selected excluding pregnant patients and patients below $15 \mathrm{yrs}$ and above $55 \mathrm{yrs}$. All patients were subjected to Transvaginal-ultransonography, saline infusion sonography and dilation and curettage (D\&C) along with endometrial biopsy. All histopathological samples were stained with Hematoxylin and Eosin stain (H\&E stain) and subsequently immunostaining was done for ER, PR and HER-2 / NEU.

Result: $37.20 \%$ cases of AUB were observed in the age group of 41-45. 0.7\% among age group of 51-55 yrs. $64.3 \%$ of rural population \& $67.2 \%$ of patients from lower socio-economic group had AUB. $48.6 \%$ cases presented with menorrhagia and $42.9 \%$ cases had etiological features of dysfunctional uterine bleeding (DUB), 20\% had endometrial polyp and 14 cases out of 140 had endometrial carcinoma. In the present study ER were positive in $84.7 \%$ and PR were positive in $76.7 \%$. HER-2/NEU expression in this study showed different histopathological pattern of endometrium in AUB. The present study also showed HER-2/NEU expression in $21.4 \%$ cases of different grades of Endometrial Carcinoma and all DUB cases showed negative HER-2 / NEU expression.

Conclusion: In this study clinical presentation, use of different diagnostic modalities and histopathological correlation were reviewed. Large number of patients belonged to age group 41-50 yrs. The incidence of AUB was maximum in rural areas and most patients belong to lower socio-economic group. By use of Hormone receptors expression of ER \& PR and observing the over expression of HER-2 / NEU the prognosis of endometrial lesions can be assessed for early and proper management.
\end{abstract}

Keywords: ER, PR, HER-2 / NEU, Imaging Devices, Dilation and Curettage

\section{Introduction}

Abnormal uterine bleeding (AUB) may be defined as any variation from normal menstrual cycle including alteration in its regularity, frequency of menstrual bleeding, duration of flow and amount of blood loss. AUB can occur at any age during reproductive period involving organic, systemic and hormonal response. Various organic lesions like fibromyoma, adenomyosis, endometrial polyp, ovarian tumours, pelvic inflammatory disease (PID), endometrial hyperplasia, endometrial carcinoma and hormonal imbalance like hypothyroidism and hypothalamic pituitary diseases can cause AUB.

AUB interferes women's physical, social and emotional quality of life. Many women also experience debilitating symptoms. Key to successful management depends largely to identify the causative factors through clinical examination, imaging devices and histopathological examination. The Human endomatrium expresses ER (Estrogen Receptor) and PR (Progesterone Receptor). The HER2 / NEU oncogene is one of the most frequently altered genes in human cancer. ER and PR over expression is found in endometrial polyps indicating the hormone receptors contribute to endometrial polyp formation ${ }^{[1-3]}$. Decrease in receptor activity is found in atypical hyperplasia and less sensitivity to progesterone therapy ${ }^{[4]}$ ER \& PR expression are generally decreased in endometrial carcinoma ${ }^{[5]} \mathrm{HER}-2$ / NEU oncogene expression demonstrated as cell membrane staining has heterogeneous expression in hyperplastic and neoplastic epithelial cells but over expression suggests 
higher grade endometrial carcinoma and is a reliable prognostic indicator ${ }^{[6]}$.

To study various actiopathological factors responsible for AUB. To correlate clinical presentation with histopathological studies along with hormone receptor markers ER, PR and markers of oncogene HER-2 / NEU for early diagnosis and prognostication.

\section{Materials and Methods}

This study was conducted during the period September 2012 to January 2015 . In this study 140 cases between 15 55 years of age group were randomly selected excluding pregnant patients and patients below 15 yrs and above 55 years, and patients and who did not give consent. This study was approved by Institutional Ethics Committee as written consent was taken from the participants.

Relevant clinical data regarding age, pattern of bleeding, amount of blood loss and use of any exogenous hormones were taken. All patients were subjected to Transvaginal ultra-sonography, saline infusion sonography and D \& C along with endometrial biopsy. All D \& C, endometrial biopsy and Hysterectomy specimen were initially stained by $\mathrm{H} \& \mathrm{E}$ stain after following standard grossing and processing procedures. Immuno stains to study ER, PR and HER 2 / NEU status were done by scheduled staining procedure and interpretation was made by following guidelines by Allred scoring system.

\section{Result}

In our study 52 out of 140 patients of AUB belong to age group of 41-45 (37.2\%), 49 patients of Para $2\left(\mathrm{P}_{2}\right)$ had AUB (35\%) and $\mathrm{P}_{4} \&$ above only 32 cases $(22.9 \%)$. History of DUB found in $42.9 \%(60 / 140)$ of patients), where as endometrial carcinoma (Gr I, II and III) found in 12 patients $(8.57 \%$ ) (Table 1$)$. Diagnosis accuracy by ultrasonography was $86 \%$ where as in Histopathological examination the accuracy was $100 \%$. Among other cases fibromyoma found in $35.9 \%$ cases and adenomyosis in $16.5 \%$ of cases. Surgical management was done as myomectomy $12.6 \%$ polypectomy $4.9 \%$, NDVH $62.2 \%$ and TAH 9.7\%. Positive expression of ER was found in $84.7 \%$ cases of proliferative, secretory endometrium where as ER expression decreased in simple hyperplasia, complex hyperplasia and endometrial carcinoma by $65 \%$, $71.4 \%$ and $64.3 \%$ respectively. PR expression is almost $100 \%$ in benign and inflammatory conditions where as decreases in endometrial carcinoma (35.7\%) HER-2 / NEU expression is 0 to $1^{+}$intensify in inflamatory as well as benign conditions where as intensity increases to $3^{+}$in endometrial carcinoma (Figure $1-8)$.

Table 1: Histological Pattern in AUB Patients.

\begin{tabular}{|c|c|c|}
\hline \multicolumn{3}{|c|}{ Histological Pattern in AUB Patients } \\
\hline Proliferative Endometrial & 12 & $8.5 \%$ \\
\hline Secretary Endometrial & 18 & $12.8 \%$ \\
\hline Exogenous hormonal effect & 17 & $12.1 \%$ \\
\hline Atrophic endometrial & 9 & $6.4 \%$ \\
\hline Chronic endometritis & 6 & $4.28 \%$ \\
\hline Simple hyperplasia without atypia & 20 & $14.2 \%$ \\
\hline Complex hyperplasia without atypia & 14 & $10 \%$ \\
\hline Endometrial Polyp & 30 & $21.4 \%$ \\
\hline Endometrial Carcinoma Gr I & 9 & $6.4 \%$ \\
\hline Endometrial Carcinoma Gr II & 3 & $2.1 \%$ \\
\hline Endometrial Carcinoma Gr III & 2 & $1.4 \%$ \\
\hline Total & 140 & $100 \%$ \\
\hline
\end{tabular}




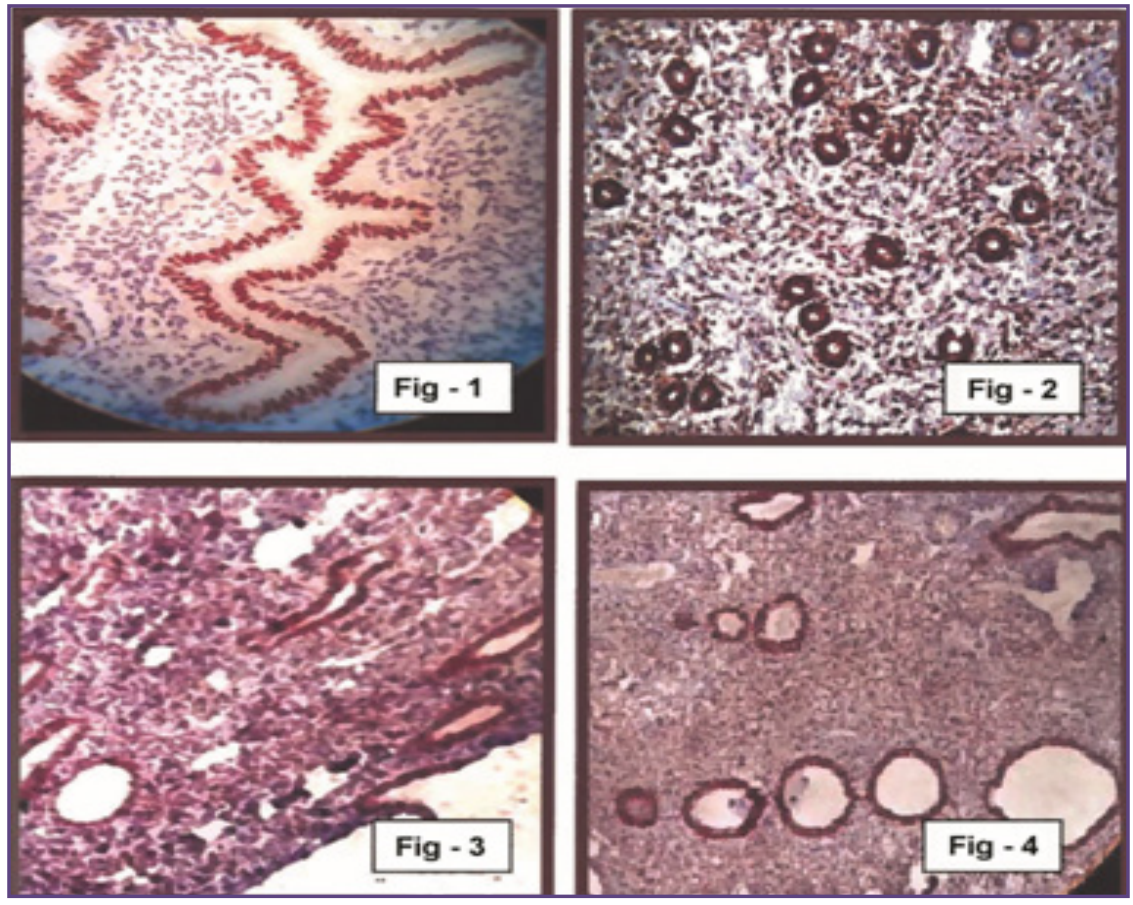

Fig. 1: Immunohistomorphology of Secretory endometrium (Positive ER expression 10x-40x); Fig. 2 : Immunohistomorphology of Proliferative endometrium (Positive ER expression 10 x 10x); Fig. 3 : Immunohistomorphology of Atrophic endometrium (Positive ER expression 10 x 10x); Fig. 4 : Immunohistomorphology of Endometrial polyp (Positive ER expression 10 x 10x).

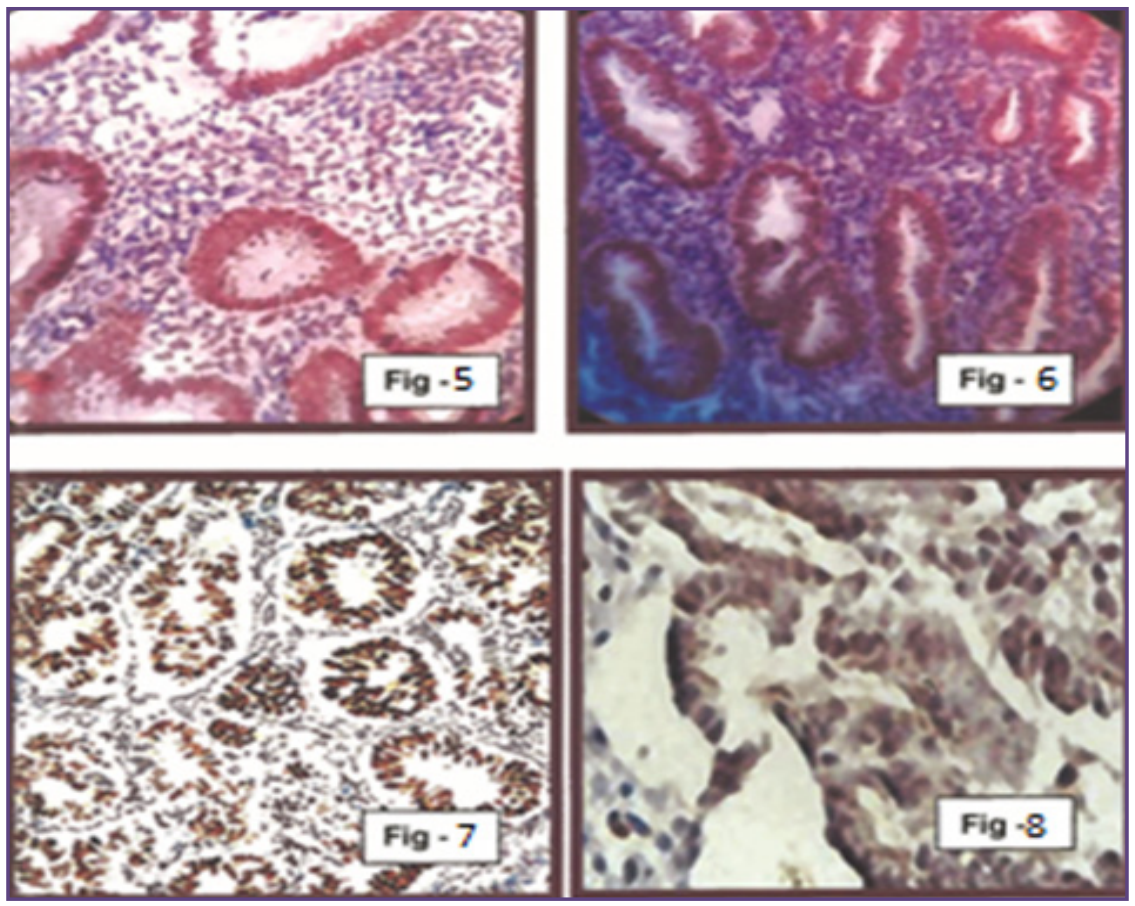

Fig. 5 :Immunohistomorphology of Simple hyperplasia without atypia (Positive ER expression 10 x 40x);

Fig. 6 : Immunohistomorphology of Complex hyperplasia without atypia (Positive ER expression 10 x 40x);

Fig. 7: Immunohistomorphology of endometrial carcinoma grade I (Positive ER expression $10 \times 40 x$ );

Fig. 8 : Immunohistomorphology of endometrioid carcinoma grade II (Positive ER expression 10 x 40x). 


\section{Discussion}

Endometrial carcinoma is the third most common malignancy among women in Southeast Asia ${ }^{[7]}$. The disease prognosis is based on several uterine and extra-uterine factors that include many molecular markers. The steroid hormones, estrogen and progesterone, play a important role in the pathogenesis of endometrial carcinoma, particularly the endometrioid variant. The decreased expressions of estrogen receptor (ER) and progesterone receptor (PR) are observed in invasive tumors with increase in both grade and stage compared to atypical hyperplasia. Hence absence of ER and PR expression may be important in the progression of endometrial carcinogenesis ${ }^{[8]}$. The age distribution of AUB in our study showed $37.9 \%$ of patients belonged to $5^{\text {th }}$ decade (41-50 yrs) where as Sunith et al. ${ }^{[9]}$ reported 33.5\% and Abdullah et al. ${ }^{[10]}$ showed 33.1\%. Many previous studies had reported the prevalence of abnormal intrauterine bleeding in patients had a median

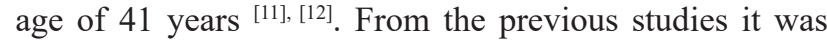
also observed that the abnormal uterine bleeding in young women was commonly misinterpreted as dysfunctional bleeding, which could lead to a delay in diagnosis ${ }^{[13]}$. In our study most patients belonged to second para about $35 \%$ where as Archana et al. ${ }^{[14]}$ reported the similar cases as $32.8 \%$. In our study commonest presenting symptom was menorrhagia $(48.6 \%)$ Khare et al. ${ }^{[15]}$ reported this to be $48.8 \%$ and Amando et al. ${ }^{[16]}$ reported this to be $47.2 \%$ DUB is commonest etiological factor (42.9\%) in our study which almost matches the report of Archana et al. ${ }^{[14]}$ (43.85\%). Some studies had reported that the etiological factors like use of contraceptive pills for one to five years, familial history of Lynch syndrome ${ }^{[12]}$. A variety of comorbid conditions are implicated in the pathogenesis of endometrioid carcinomas, including diabetes mellitus, obesity, hypertension and other organ malignancies [17]. Histopathological findings show endometrial polyp $(21.4 \%)$ followed by simple hyperplasia without atypia (14.2\%) complex hyperplasia without atypia in $10 \%$ cases and endometrial carcinoma in $10 \%$ cases. Our study correlates with studies undertaken by Mirza et al. ${ }^{[18]}$ and Ejaz et al. ${ }^{[19]}$. Incidence of ER and PR expression in endometrial polyps in present study is $86.7 \%$ and $73.3 \%$ which correlates with study of Armando et al. ${ }^{[16]}$.

Incidence of positive expression of ER in endometrial hyperplasia is $71.4 \%$ and Endometrial Carcinoma Gr I (66.7\%), Gr II (66.7\%), Gr III 50\% which is at par with study of Cai et al. ${ }^{[20]}$. PR expression in Endometrial hyperplasia is $60 \%$ and endometrial Carcinoma Gr I (44.4\%), Gr II (33.3\%) and Gr III (00\%) in present study which matches with the study of Bergeron et al. ${ }^{[21]}$. HER 2 / NEU expression in present study in endometrial hyperplasia is $35.3 \%$ and endometrial carcinoma $\mathrm{Gr}$ I (11.1\%), Gr II (33.3\%) and Gr III 50\% which is at par with study of Rasty et al. ${ }^{[22]}$ which concludes that HER 2 / NEU expression increases significantly in high grade endometrial carcinoma. From the literature it was found that hormone receptors are positive in 35\%-90\% of endometrial carcinomas and the absence of these receptors might indicate advanced disease. The current investigations has reinforced the fact that hormone receptor expression should be studied in endometrioid carcinomas as they have prognostic implications. Receptor negative patients need regular follow up visits, which will aid in earlier detection of recurrence and metastasis.

\section{Conclusion}

In this study the clinical presentation, diagnosis modalities along with histopathological correlation have been reviewed along with IHC of endometrial biopsy to find the status of ER, PR and HER 2 / NEU expression. Most common organic pathology after surgical treatment was fibromyoma. Maximum cases of AUB were managed with NDVH, Myomectomy, Polypectory which are ER and PR expression were high almost in all cases of DUB and in benign lesions, where as decreased in high grade endometrial carcinoma HER-2 / NEU over expression was associated with high grade Endometrial Carcinoma. So negative HER-2 / NEU expression is a good prognostic indicator. Lesions with positive ER, PR can be treated with hormone therapy and radical surgery can be avoided. ER, PR and HER-2 / NEU are very important immunemarkers and are helpful to assess the prognosis as well as to avoid the unnecessary surgery for the patients who can be medically managed.

\section{Abbreviations and symbols}

AUB: Abnormal Uterine Bleeding

ER: Estrogen Receptor

PR: Progesterone receptor

DC: Dilation and curettage

H \& E: Hematoxylin and Eosin stain

DUB: Dysfunctional uterine bleeding

PID: pelvic inflammatory disease

\section{Declarations}

The study was not supported by any funds and grants

The authors declared "No conflict of interest"

\section{References}

1. Elza C, De Almeidaa SA, Alberto A, Nogueiraa F et al. Immunohistochemical expression of estrogen and progesterone receptors in endometrial polyps and adjacent endometrium in postmenopausal women. Maturitas. 2004; 49 : 229-233. 
2. Mc Gurgan P, Taylor LJ, Duffy SR, O' Donovan PJ. Are endometrial polyps from premenopausal woman similar to postmenopausal women. Maturites. 2006; 54 : 277-284.

3. Lopes RG, Baracat EC, de Albuquerque Neto LC, Ramos JF, Yatabe S, Depesr DB, Lippi UG. Analysis of oestrogen and progesterore receptor expression in endometrial polyps. J. Mirimum invasive Gynecol. 2017; 14 : 300-303.

4. Teleman S, Hilgarth $\mathrm{M}$, Freudenberg $\mathrm{N}$, Bettendorf $\mathrm{H}$, Mihailovici MS. An immunohistochemical study of the steroid hormone receptors in endometrial hyperplasia. Rev Med Chir Soc Med Nat Iasi. 1999; 103(1-2):138-41.

5. Nunobiki O, Taniguchi E, Ishii A, Tang W, Utsunomiya $H$, Nakamura Y, Mori I, Kakudo K. Significance of hormone receptors status and tumor vessels in normal hyperplastic and neoplastic endometrium. Pathology Int. 2003; 53 (12) 846-52.

6. Rasty G, Richard M, Leo Lu, Paul K, Guy B, Shahla M. Expression of HER-2 / NEU oncogene ia normal, hyperplastic and malignant endometrium. Ann Clin. Lab Sci. 1998; $28: 138-143$.

7. Kim JW, Kim SH, Kim YT, Kim DK. Clinicopathologic and biological parameters predicting the prognosis in endometrial cancer. Yonsei Med J. 2002; 43(6): 769-778.

8. Stoian SC, Simionescu C, Mărgăritescu, Stepan A, Nurciu M. Endometrial carcinomas: correlation between ER, PR, Ki67 status and histopathological prognostic parameters. Rom J Morphol Embryol. 2011; 52(2):631-6.

9. Sunitha $\mathrm{CH}$. Clinical study of diagnostic hysteroscopy in AUB and its histopathological correlation (dissertation). Bijapur: SHRI B.M. Patil medical college hospital \& research centre; 2006.

10. Abdullah LS, Bondagji NS; Histopathological Pattern of Endometrial Sampling Performed for Abnormal Uterine Bleeding. Bahrain Med Bull. 2011; 33(4): 1-6.

11. Hanprasertpong J, Sakolprakraikij S, Geater A. Endometrial cancer in Thai women aged 45 years or younger. Asian Pac J Cancer Prev. 2008; 9: 58-62.
12. Richter CE, Qian B, Martel M, et al. Ovarian preservation and staging in reproductive-age endometrial cancer patients. Gynecol Oncol. 2009; 114: 99-104.

13. Pellerin GP, Finan MA. Endometrial cancer in women 45 years of age or younger: a clinicopathological analysis. Am J Obstet Gynecol. 2005; 193: 1640-4.

14. Archana B and Fonseca M. Evaluation and histopathological correlation of abnormal uterine bleedia in premenopausal women Bombay Hosp. J. 2010; 52 : 69-72.

15. Khare A, Bansal R, Sharma S, Elhence P, Makkar N, Tyagi Y. Morphological spectrum of endometrial in patients presenting with DUB. Peoples Journal of scientific research. 2012; 5(2) : 13-16.

16. Armando A, Jose V, Anderson P, Rogério L, Aarão M, Pinto N, Lucia costa P. Immunohistochemical expression at ER, PR receptors in endometrial polyp. Maturities. 2015; 49 : 229-233.

17. Williams GP. The role of oestrogen in the pathogenesis of obesity, type 2 diabetes, breast cancer and prostate disease. Euro J Cancer Prev. 2010; 19(4): 256-71.

18. Mirza T, Saadia A, Aamir M, Sadiya A, Tariq M, Tazeen M. Histopathological pattern of AUB. J of Basics and Applied Sciences, 2012; 8: 14-17.

19. Ejaz S. Causes of post menopausal bleeding Cancer. J. Clin. 2001; 4: 260-262.

20. Cai Q. Expression of ER receptors alpha and beta protein in endometrial carcinoma. cancer therapy. 2003; 6: 907-912.

21. Bergeron C, Ferenczy A, Toft DO. Immunohistochemical study of PR receptors during menstrual cycle. Lab. Invest. 1988; 59: 862-869.

22. Rasty G, Murray R, Lu L, Kubilis P, Benrubi G, Masood S. Expression of HER-2/neu oncogene in normal, hyperplastic, and malignant endometrium. Ann Clin Lab Sci. 1998; 28(3):138-43

*Corresponding author:

Dr TanushreeSatpathy, Assistant Professor, Dept. of Pathology, GITAM Institute of Medical sciences and research, Gitam university

Vishakhapatnam-530045 INDIA

Email: jyothipgis@gmail.com, tanu_sat@yahoo.com

Financial or other Competing Interests: None. 